\title{
Experimental pain in the stomach: a model based on electrical stimulation guided by gastroscopy
}

\author{
A M Drewes, L Arendt-Nielsen, J H Jensen, J B Hansen, H B Krarup, U Tage-Jensen
}

\begin{abstract}
Background-Abdominal pain is often variable in intensity and difficult to characterise due to its referred pain pattern. Clinical pain is furthermore confounded by various emotional and cognitive factors.
\end{abstract}

Aims-To develop and apply an experimental model to induce localised gastric pain.

Subjects-Twelve healthy male volunteers.

Methods-Stimulating electrodes were mounted on a biopsy forceps and electric stimuli were delivered during gastroscopy. Single, five repeated, and continuous stimuli were given at four locations in the stomach. Pain detection thresholds and pain intensities were assessed together with localisation of the referred pain area. Results-Pain detection thresholds were higher in the prepyloric region compared with those obtained at the lesser and greater curvature. Increasing stimulus intensity resulted in augmented pain perception and repeated stimuli elicited pain at a lower stimulus intensity than single stimuli. Continuous stimuli evoked constant $(33 \%)$, increasing (33\%), or decreasing $(33 \%)$ pain. The localisation of referred pain varied considerably in the subjects.

Conclusions-The model seems relevant to study basic pain mechanisms elicited by localised stimuli in the stomach. The experimental data support the premise that a gastric focus should always be suspected in patients referred with different kinds of abdominal pain.

(Gut 1997; 41: 753-757)

Keywords: experimental pain; gastric; stimulation; temporal summation; referred pain

Abdominal pain represents a clinical challenge in gastroenterology. Information on pain onset, intensity, and localisation are often the major guidelines to a clinical diagnosis with consequences for treatment. Visceral pain is different from cutaneous pain, being diffuse, and often accompanied by referred pain and autonomic responses as well as cutaneous hyperalgesia. ${ }^{12}$ Typical pain in different gastrointestinal diseases is described in various textbooks. For example, gastric pain due to peptic ulcer disease is characterised as a burning or gnawing pain located sharply focal in the midepigastrium, whereas symptoms such as burning discomfort behind the sternum are thought to be caused by associated oesophageal reflux of acidic stomach contents. ${ }^{3}$ It is, however, well known that gastric pain can be atypical with referred pain to various places in the abdomen. Also, the size of the pathological lesion often does not correspond with the pain intensity, as in ulcer disease, for example. Furthermore, in the clinical situation description of pain is confounded by various factors such as emotional, cognitive, and social elements and is thus difficult to assess. Therefore, valid experimental pain models are needed in order to study the physiology of nociceptive inputs to the gastric mucosa. However, most models of visceral pain are either limited to anaesthetised animals or biased by the lack of visual monitoring during stimulation. The aims of the current paper were to describe a new technique to elicit experimental pain in localised regions of the human stomach and to draw parallels to clinical situations.

\section{Methods}

SUBJECTS

Twelve healthy men, median age 22 years (range 20-24), volunteered to participate in the study. All were compensated for their assistance. None had a history of previous gastrointestinal disease and they had no gastrointestinal complaints. The study was conducted according to the Helsinki Declaration and approved by the local ethics committee.

\section{ELECTRICAL STIMULATION}

Stimulating electrodes (platinum balls with a 1 $\mathrm{mm}$ diameter) were mounted on a biopsy forceps on the gastroscope (fig 1). The electrodes were embedded into a plastic coating and connected by Teflon coated stainless steel wires to the electrical stimulator unit. During stimuli the jaws of the forceps were opened giving a distance of $4 \mathrm{~mm}$ between the electrodes. The constant current stimuli were controlled by a computer and were independent of the electrode impedance (Aalborg University, Denmark). The intensity of the current was limited to $80 \mathrm{~mA}$ and the voltage to 200 V. Five constant current pulses (rectangular with a duration of $1 \mathrm{~ms}$ ) applied at $200 \mathrm{~Hz}$ were defined as a "single stimulus". Five of these stimuli given consecutively at $2 \mathrm{~Hz}$ were defined as "repeated stimuli". For "continuous stimulus" the single stimulus was delivered at 4 $\mathrm{Hz}$ for a maximum of two minutes. Subjects were instructed to indicate manually when the single and repeated stimuli could be felt (sensation threshold, ST) and when pain was 


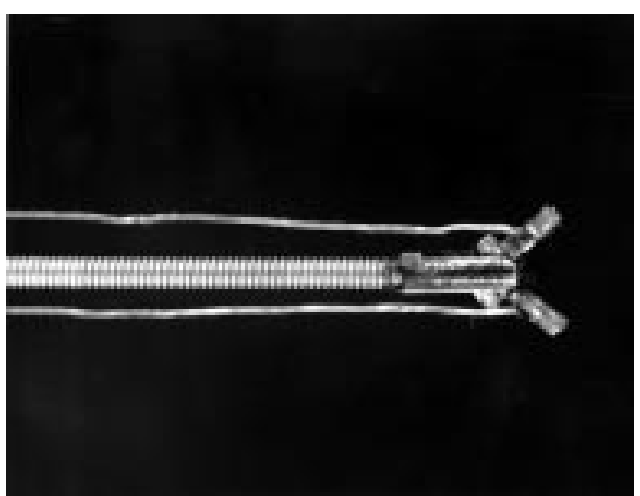

Figure 1: The stimulator unit for the gastroscope showing the biopsy forceps mounted with platinum ball stimulation electrodes. During stimulation the stimulator was pressed against the mucosa under visual inspection.

perceived (pain detection threshold, PDT). The intensity was gradually increased in steps of $1 \mathrm{~mA}$ during an interactive procedure until these thresholds were reached. To assess further levels of pain intensity a stimulus response curve was determined using a $0-10$ $\mathrm{cm}$ electronic visual analogue scale (VAS) ranging from $0=$ no pain to $10=$ unbearable pain. The intensity of the VAS was displayed for the subject simultaneously with the scoring which could be done manually on the device. Five stimuli with increasing intensity were given to obtain pain levels ranging from approximately 1 to 5 on the VAS. For the session with continuous stimulation, a current intensity giving approximately 2.5 on the VAS during the repeated stimulus was given. Pain was scored on the VAS simultaneously with the continuous stimulation. If pain increased above 7 on the VAS the stimulation was stopped. The subject could activate a button which immediately stopped the stimulations. The total duration of the experiment was approximately 40 minutes.

PROTOCOL

A venous line was established and for security continuous monitoring of pulse and blood pressure was performed (Pro-paq 102, Protocol Systems Inc., Oregon, USA). The subjects fasted for eight hours and underwent gastroscopy (Olympus GIF-100, Hamburg) with minimal air insufflation. No analgesics or sedatives were given during the procedure. After a few minutes the subject was accustomed to the procedure and the stimulator was inserted into the biopsy channel of the scope. The tip of the forceps was pressed firmly against the mucosa in the regions studied under continuous visual inspection. After the area for stimulation was localised, nearly all the air was sucked out of the stomach. Stimuli were given in four areas: prepyloric region; lesser curvature near the angular notch; major curvature near the angular notch; and duodenal bulb. Characteristic anatomical areas were identified so that if peristaltic movements displaced the electrode during the procedure it could easily be repositioned. During each stimulus session the subject was told to indicate on a standardised drawing of a human body where the pain was felt and the corresponding area of referred pain was pointed out and referred to the drawing. For calculation of the relative areas of referred pain, the mean of the largest and smallest diameters (in $\mathrm{mm}$ on the drawings) perpendicular to each other was used.

In the order described below, the following procedures were performed:

(1) For single and repeated stimuli in the prepyloric area the ST and PDT were determined. Based on five stimuli given with increasing intensity ranging from approximately 1 to 5 on the VAS the stimulus response function was found. Continuous stimuli were given in this region.

(2) At the greater and lesser curvature and in the duodenal bulb the PDT was determined for repeated stimuli.

(3) For repeated stimuli in the prepyloric area the PDT was determined again and the five stimuli used at the first test were again given with increasing intensity while the subject scored the corresponding pain level on the VAS.

After the study subjects were questioned further regarding the pain during stimuli and any side effects (such as nausea, oppression, etc), and the Danish version of the McGill Pain Questionnaire $^{4}$ was completed for the pain elicited during continuous stimulation.

STATISTICAL ANALYSIS

Wilcoxon's rank sum test was used for paired comparisons. For the stimulus response curves linear regression was performed and in the test retest calculations Spearman's test was used. A value of $p$ less than 0.05 was regarded as significant.

\section{Results}

All subjects felt pain with stimuli at and above the pain detection threshold and the pain stopped immediately after cessation of the stimuli. Pain was typically described as an acute unpleasant, dull, and colic-like sensation. No nausea or other concomitant feelings were experienced. No subjects had pain or any other side effects after the study. No cardiovascular side effects were observed. The most frequent words from the McGill Pain Questionnaire used to describe the pain were boring (58\%), sharp (50\%), annoying (50\%), tingling (50\%), shooting $(42 \%)$, and taut $(42 \%)$. Within the sensory dimension of the questionnaire, ${ }^{4}$ all chose some descriptors. Eight used words from the affective group, and 11 and nine subjects respectively, chose words from the evaluative and miscellaneous groups to describe their pain. No systematic correlations between the descriptors chosen and the pain intensity or the area of pain referral described below were found.

PREPYLORIC REGION

Table 1 presents results from the stimulations. Median sensation threshold was similar for single and repeated stimuli $(\mathrm{p}=0.083)$, whereas the repeated stimuli resulted in the lowest pain detection threshold $(p=0.023)$. The areas of 
TABLE 1 Current intensities $(m A)$ used for eliciting sensation or pain thresholds in the various regions for the 12 subjects

\begin{tabular}{|c|c|c|c|c|c|c|c|c|}
\hline \multirow[b]{2}{*}{ Subject no } & \multicolumn{2}{|c|}{$\begin{array}{l}\text { Prepyloric region single } \\
\text { stimuli }\end{array}$} & \multicolumn{2}{|c|}{$\begin{array}{l}\text { Prepyloric region } \\
\text { repeated stimuli }\end{array}$} & \multirow{2}{*}{$\begin{array}{l}\text { Prepyloric } \\
\text { retest } \\
\text { PDT }\end{array}$} & \multirow{2}{*}{$\begin{array}{l}\text { Lesser } \\
\text { curvature } \\
\text { PDT }\end{array}$} & \multirow{2}{*}{$\begin{array}{l}\text { Major } \\
\text { curvature } \\
\text { PDT }\end{array}$} & \multirow{2}{*}{$\begin{array}{l}\text { Duodenal } \\
\text { bulb } \\
\text { PDT }\end{array}$} \\
\hline & $S T$ & $P D T$ & $S T$ & $P D T$ & & & & \\
\hline 1 & 11 & 13 & 8 & 10 & 8 & 5 & 5 & 6 \\
\hline 2 & 3 & 20 & 8 & 28 & 20 & 9 & 5 & 20 \\
\hline 3 & 20 & 21 & 13 & 20 & 5 & 3 & 12 & 15 \\
\hline 4 & 20 & 22 & 10 & 12 & 15 & 15 & 15 & 15 \\
\hline 5 & 8 & 25 & 4 & 10 & 18 & 5 & 5 & 15 \\
\hline 6 & 4 & 13 & 4 & 7 & 1 & 4 & 2 & 2 \\
\hline 7 & 3 & 4 & 5 & 5 & 2.5 & 3 & 3 & 3 \\
\hline 8 & 8 & 25 & 2 & 15 & 15 & 4 & 1.5 & 2.5 \\
\hline 9 & 3 & 6 & 4 & 5 & 8 & 1 & 1 & 2 \\
\hline 10 & 2 & 4 & 1 & 2 & 5 & 1.5 & 1.5 & 3.5 \\
\hline 11 & 7 & 13 & 1.5 & 4 & 6 & 2.5 & 2 & 3 \\
\hline 12 & 1 & 16 & 1 & 10 & 15 & 6 & 8 & 10 \\
\hline Median & 5.5 & 14.5 & 4 & 10 & 8 & 4 & 4 & 4.75 \\
\hline
\end{tabular}

Repeated stimuli were five single pulses given at $2 \mathrm{~Hz}$. The retest procedure was a reiteration of the repeated stimulation in the prepyloric area.

ST, sensation threshold; PDT, pain detection threshold.

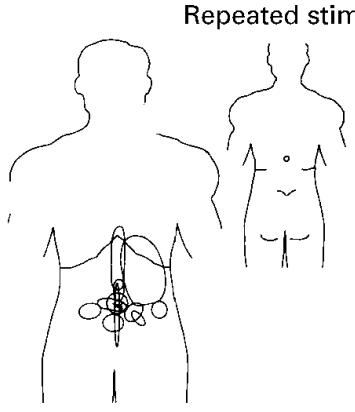

Pre-pyloric

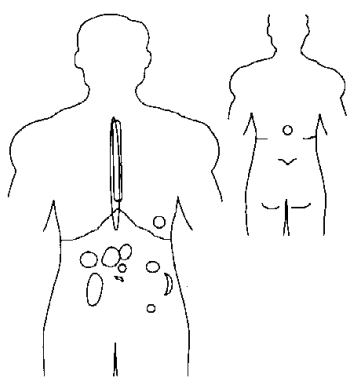

Lesser curvature

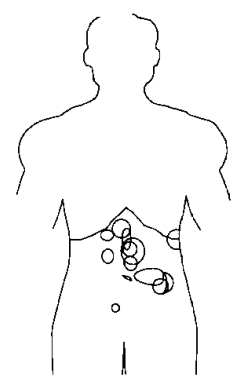

Greater curvature

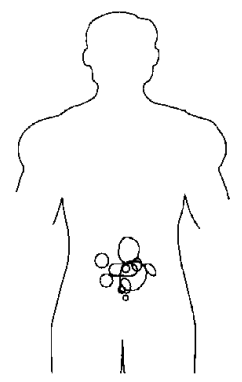

Duodenum

Figure 2: The areas of referred pain for all subjects during repeated electrical stimuli at: (a) the prepyioric region; (b) the greater curvature; (c) the lesser curvature; (d) the duodenal bulb.

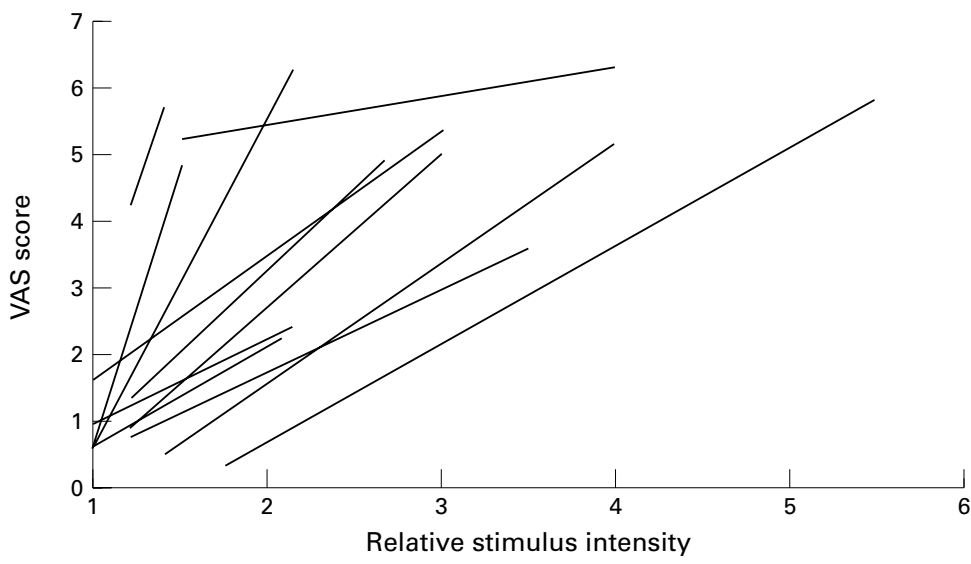

Figure 3: Linear regression lines for the first stimulus response experiment in the prepyloric region using repeated electrical stimuli. The $x$ axis shows stimulus intensity relative to intensities at the pain detection threshold.

referred pain varied between subjects (fig 2). No systematic differences in site of referred pain comparing single and repeated stimuli were found. During the continuous stimuli all VAS ratings followed a waxing and waning curve over the stimulation period. Generally, four subjects rated increasing pain intensity on the VAS, in four subjects the pain was constant, and in the remaining four it gradually decreased. The relative area of referred pain was greater during continuous and repeated stimulation compared with single stimuli (9.5 (3-20) and $6.75(3-21)$ versus 6.25 (2.5-8.5), $\mathrm{p}=0.039 ; \mathrm{p}=0.009)$. A trend towards a significant correlation between the pain detection thresholds obtained at the test retest experiment was found (Spearman's test; $p=0.05$ ). The stimulus response curves for increasing stimulus intensities were nearly linear. In fig 3 the ordinary linear regression estimates for the individual lines during repeated stimuli are shown. The median slopes for the stimulus response curves were for single stimuli 1.8 (0.24-9.77), and for repeated stimuli 1.85 (0.44-8.52). No differences between these slopes were found $(p=0.33)$.

CURVATURES AND DUODENAL BULB

The pain detection thresholds for repeated stimuli were significantly smaller at the lesser and major curvatures compared with the prepyloric region $(\mathrm{p}=0.008$ and $\mathrm{p}=0.007$ respectively). At the lesser curvature the pain was spread to various regions ranging from the right inguinal region to the back and two subjects reported pain in the retrosternal region (fig 2). Contrary to the prepyloric stimuli only three felt pain in the periumbilical area. For the retrosternal pain it was described as heartburn. Pain thresholds for the duodenal bulb were similar to those in the prepyloric region $(p=0.1)$ and stimuli gave pain referred to the periumbilical region.

\section{Discussion}

A model for experimental stomach pain was developed and applied. Using the model we were able to elicit pain as a result of acute stimuli in various regions of the stomach mucosa mimicking, for example, ulcer disease. The stimulus response curves showed increas- 
ing pain level for increasing stimulus intensity. The decreased pain thresholds for repeated compared with single stimuli probably indicated the importance of temporal summation shown in other cutaneous and visceral models. ${ }^{56}$ The different current intensities necessary to elicit pain in the four regions could reflect the regional variations in pain sensitivity - that is, innervation density. During continuous stimulation the waxing and waning of pain intensity may be a result of modulation or plasticity of the enteric nervous system. The propagation of referred pain depended on the type of stimulus modality. Finally, the area of referred pain varied considerably between different subjects, which may parallel clinical situations.

Several models of experimental visceral pain exist. Traditionally these are divided into mechanical, chemical, ischaemic, thermal, and electrical stimulus modalities. ${ }^{78}$ The mechanical stimuli have been most widely used as they are thought to be more natural and selective. ${ }^{79}$ For gastric stimulation, however, diseases such as peptic ulcer are more localised and do not usually result in distension of the organ. Therefore, discrete stimuli seem most appropriate in this region. Chemical stimuli have so far only been of limited value in human studies ${ }^{2}$ and thermal stimuli probably do not elicit pain. ${ }^{8}$ Finally, ischaemic stimuli are not acceptable in a human model. Previous studies of electrical stimuli in viscera have been limited to electrodes mounted on intraluminal catheters or tubes ${ }^{60-13}$ without the possibility of visual inspection, which in the gastric area is necessary to secure the electrode position. Furthermore, the potential hazards of insufficient electrode contact with the mucosa in these former studies is obvious and the possibility for inspection of motility does not exist. This probably could explain the varying sensations obtained as well as the great span between ST and PDT observed in these studies. $^{8} 10$

Although electrical stimuli are not specific (all afferents in the area are depolarised) the model described provides several advantages. Firstly, the stimulus can be applied distinctly in all regions of the stomach and visual monitoring of the mucosa during stimulation is possible. Secondly, stimuli can be given at well defined time intervals with controlled pulse configuration and frequency and after cessation, pain immediately stops. Thirdly, as the pain intensity increases proportionally to the current, stimulus response functions can be determined.

One of the limitations of the present study is that impedance between the electrodes was not measured. The possibility that electrode contact with the mucosa varied cannot be excluded. Also standardised sham stimuli were not delivered. However, in all subjects several stimuli with intensity below ST were given randomly during the sessions in order to test electrode-mucosa contact, and none reported pain with a stimulus intensity below the PDT. Furthermore, pressure exerted by the electrode on the gastric wall was not felt by the subjects.
Finally, during the stimulus response functions stimuli were not randomised. As stimuli above 5-7 on the VAS were very unpleasant, and we were not able to predict the stimulus intensity for which this pain level was reached, stimuli in this experiment were gradually increased. However, only when stimuli were expected to be above 5 on the VAS, were subjects asked if they could tolerate further intensity. Otherwise they were only informed to score the intensity and had no knowledge regarding the gradual increase in the stimulus. Therefore, we do not believe that response bias influenced the results. It is possible that the presence of the gastroscope modified the perception of pain. All subjects were, however, able to discriminate the discomfort induced by the scope from the distinct pain elicited by the electrical stimuli, and they were accustomed to the procedure before the stimuli started. Therefore we do not believe that the gastroscope induced major bias in the study. Finally, we sucked out nearly all the air after the stimulation area was localised to avoid distension of the stomach wall. It cannot be excluded, however, that the remaining air may somehow influence the perception due to the mechanical stimulus although the effect is probably of only minor importance.

The significant higher current intensity necessary to reach pain thresholds for single compared with repeated stimuli shows the successive increasing pain sensation to repeated stimuli known as the "temporal summation phenomenon" which has also been shown for cutaneous and visceral pain in other structures. ${ }^{6}{ }^{13-16}$ This phenomenon might parallel the "wind-up" described in animal models closely related to the hyperexcitability and plasticity of central nervous structures. The variability of the enteric nervous system was further shown in the continuous stimuli where different responses to long term stimuli occurred. The clinical implications of this observation are obvious as, for example, intermittency of pain in acute gastrointestinal diseases is a common observation despite the pathological lesion being constant. ${ }^{3}$ These observations further emphasise the fact that pain intensity may be of limited value in monitoring patients with diseases of the stomach and related regions.

The pain characteristics were similar to those reported in patients with upper abdominal diseases. Some described the pain in terms of previous known discomfort during an attack of acute gastroenteritis, whereas others described the feeling like drinking ice cold liquid. Different words from the McGill Pain Questionnaire were used which is in accordance with other studies on visceral pain. ${ }^{2}$

The different pain thresholds in the four areas parallels the clinical observation that the size of the lesion does not always reflect the symptoms felt by the patient. Parameters such as the growth potential, relation to nervous structures, as well as the acidity of the stomach contents, probably also contribute to the pain. However, given that these parameters are unaltered, the different thresholds for visceral afferents shown in this paper may also explain this 
phenomenon. The finding that one subject first experienced pain in the prepyloric area when the stimulus intensity increased to $28 \mathrm{~mA}$ (being 14 times as high as the level in the subject having the lowest threshold) may parallel the clinical situation where some patients may not feel pain at all despite having a large gastric ulcer.

The finding of most clinical interest in this stydy is that the site to which pain was attributed varied from subject to subject, even though the stimulus had been applied to the same distinct anatomical region in all of them. These findings are supported in models using mucosal stimuli of the gut, where symptoms were perceived in very different abdominal regions. ${ }^{210}$ In particular, the finding that stimuli in healthy subjects without any dyspeptic complaints may elicit typical heartburn is interesting as this symptom is thought to be associated with oesophageal or cardiac pain. The pain could not be related to reflux of stomach contents as no contractions of the mucosa were seen, as reported in previous studies $^{2}$; furthermore, the pain disappeared immediately after the stimulus ceased. Back pain was also described during stimulation, a phenomenon which is traditionally seen in structures close to the vertebrae as, for example, in pancreatic pain. These findings do not completely parallel the traditional "viscerotomes" described in the literature. ${ }^{2}$

We conclude that the model seems relevant for the study of basic physiological phenomena in gastric nociception. Evidence is provided that gastric pain in disease is not necessarily related to the size or location of a lesion. Furthermore, the great variability in the area of referred pain shown in this study emphasises the clinical experience that a gastric or duodenal lesion can be found in patients with a great variety of abdominal pain complaints.
The study was supported by the Danish National Research Foundation, the Danish Cancer Society, "Grosserer L F Foghts Fond", and "Laegernes Forsikringsforening af 1891". Lis Meyer, Aalborg Hospital, and Morten Haugland, Aalborg University, are acknowledged for technical assistance.

1 Cervero F. Visceral pain. In: Dubner R, Gebhart GF, Bond MR, eds. Pain research and clinical management. Vol 3. Proceedings of the Vth World Congress on Pain. Amsterdam: Elsevier, 1988:216-26.

2 Ness TJ, Gebhart GF. Visceral pain: a review of experimental studies. Pain 1990;41:167-34.

3 Haubrich WS. Abdominal pain. In: Haubrich WS, Schaffner F, Berk JE, eds. Gastroenterology, Vol. 1. Philadelphia: WB Saunders Company, 1995:11-29.

4 Drewes AM, Helweg-Larsen S, Petersen P, Brennum J, Andreasen A, Poulsen LH, Jensen TS. McGill Pain Questionnaire translated into Danish: experimental and clinical findings. Clin f Pain 1993;9:80-7.

5 Arendt-Nielsen L. Wind-up and plasticity: is there a correArendt-Nielsen L. Wind-up and plasticity: is there a cort
lation to clinical pain. Eur f Anaesthesiol 1995;12:1-7.

6 Frøbert O, Arendt-Nielsen L, Bak P, Funch-Jensen P, Bagger JP. Oesophageal sensation assessed by electrical stimuli ger JP. Oesophageal sensation assessed by electrical stimuli and brain evoked potentials-a

7 Gebhart GF, Ness TJ. Mechanisms of visceral pain. In: Bond MR, Chariton JE, Woolf CJ, eds. Proceedings of the VIth World Congress on Pain. Amsterdam: Elsevier, 1991: 351-63.

8 Azpiroz F. Sensitivity of the stomach and small bowel: human research and clinical relevance. In: Gebhart GF, ed. Visceral pain. Progress in pain research and management. Seattle: IASP Press, 1995:391-428.

9 Gebhart GF, Meller ST, Euchner-Wamser 1, Sengupta JN. Modelling visceral pain. In: Vecchiet L, Albe-Fessard D, Lindbiom U, Giamberardino MA, eds. New trends in referred pain and hyperalgesia. Amsterdam: Elsevier, 1993: referred pair.

10 Accarino AM, Azpiroz F, Malagelada J-R. Symptomatic responses to stimulation of sensory pathways in the responses to stimulation of sensory path
jejunum. Am f Physiol 1992;263:G673-7,

11 Accarino AM, Azpiroz F, Malagelada J-R. Selective dysfunction of mechanosensitive intestinal afferents in irritable bowel syndrome. Gastroenterology 1995;108:636-43.

12 Drewes AM, Arendt-Nielsen L, Bak P, Hansen JB, Tage-Jensen U. Electric stimulation of the ileum mucosa: a pilot study of pain physiology [abstract]. Scand F Gastroenterol 1995;30(suppl 209):PO12.

13 Arendt-Nielsen L, Drewes AM, Hansen JB, Tage-Jensen U. Plasticity of gut pain in man: an experimental investigation using short and long duration transmucosal electrical stimulation. Pain 1997;69:255-62

14 Price DD, Hu JW, Dubner R, Gracely RH. Peripheral suppression of first and central summation of second pain evoked by noxious heat pulses. Pain 1977;3:57-68.

15 Arendt-Nielsen L, Brennum J, Sindrup S, Bak P. Electrophysiological and psychological quantification of temporal summation in the human nociceptive system. Eur $\mathcal{F} A p p l$ Physiol 1994;68:266-73.

16 Ness TJ, Metcalf AM, Gebhart GF. A psychophysiological study in humans using phasic colonic distension as a noxious visceral stimulus. Pain 1990;43:377-86. 